\title{
Effect of TNFRSF6B neutralization antibody on cell growth suppression and apoptosis induction in glioma cells
}

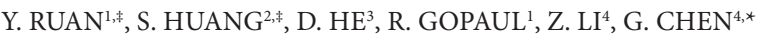 \\ ${ }^{1}$ Department of Neurosurgery, First Affiliated Hospital of Guangxi Medical University, 6 Shuangyong Road, Nanning, Guangxi Zhuang Au- \\ tonomous Region 530021, P. R. China; ${ }^{2}$ Department of Radiology, First Affiliated Hospital of Guangxi Medical University, 6 Shuangyong Road, \\ Nanning, Guangxi Zhuang Autonomous Region 530021, P. R. China; ${ }^{3}$ Department of Neurology, Seventh Affiliated Hospital of Guangxi Medical \\ University, Wuzhou Worker Hospital, Wuzhou, Guangxi Zhuang Autonomous Region 530021, China; ${ }^{4}$ Department of Pathology, First Affliated \\ Hospital of Guangxi Medical University, 6 Shuangyong Road, Nanning, Guangxi Zhuang Autonomous Region 530021, P. R. China
}

*Correspondence: chen_gang_triones@163.com

${ }^{*}$ Contributed equally to this work.

Received September 18, 2014 / Accepted February 16, 2015

\begin{abstract}
Previously, we have reported that tumor necrosis factor receptor super-family member 6b (TNFRSF6B) is overexpressed in glioma tissues, as well as in the supernatant of cultured glioma cells. However, the function of TNFRSF6B in glioma remains unclarified. The aim of the study was to investigate the effect of anti-TNFRSF6B neutralization monoclonal antibody on the malignant phenotype of glioma cells in vitro. Three glioma cell lines U251MG, LN-308 and U87MG were treated with anti-TNFRSF6B antibody. Cell growth, cell cycle, apoptosis and relevant downstream signaling were detected further. We found that anti-TNFRSF6B antibody inhibited cell growth and induced apoptosis in time and dose-dependent manners in the glioma cells tested. Cell cycle was also arrested in G1/S-phase. Furthermore, anti-TNFRSF6B antibody downregulated the level of MMP-2, phospho-ERK1/2 and phospho-AKT. In conclusion, antiTNFRSF6B treatment might be a critical targeted therapy strategy for gliomas due to its contribution to suppress cell growth and induce apoptosis.
\end{abstract}

Key words: TNFRSF6B, glioma, proliferation, apoptosis, neutralization antibody

Malignant brain tumors are one of the most related causes of morbidity and mortality across a wide range of individuals [1]. Malignant gliomas, i.e., anaplastic astrocytoma and glioblastoma, are particularly aggressive [2]. They are the most frequent class of primary malignant brain tumors in China $[3,4]$. Many studies on this subject have produced advances in the understanding of glioma biology and pathogenesis and to the improvement of new agents for targeted molecular therapy [5]. However, the median survival of patients with newly diagnosed malignant glioma is only 12-14 months despite advanced therapeutic strategies. Thus, there is an urgent need to discover new biomarkers for the early detection and targeted molecular therapy. Tumor necrosis factor receptor super-family member $6 \mathrm{~b}$ (TNFRSF6B, also termed as DcR3) locates on 20q13 [6-9] and we have previously reported the overexpression of TNFRSF6B mRNA and protein in sera or tissues of several human malignancies [10-13], including glioma [14]. We found that TNFRSF6B protein was significantly upregulated in glioma tissues, as well as in the supernatants of glioma cell lines and freshly primary cultured glioma cells. TNFRSF6B expression also had a positive correlation with tumor pathological grade. However, the exact role and pathomechanism of TNFRSF6B on the malignant phenotypes of glioma remains unclarified. Previously, our work showed that anti-TNFRSF6B neutralization monoclonal antibody could inhibit cell proliferation, reduce apoptosis and restrain migration ability in hepatocellular carcinoma HepG2 cells. Based on the overexpression of TNFRSF6B in glioma, we speculated that anti-TNFRSF6B neutralization monoclonal antibody could also affect the function of glioma cells. Therefore, in the present study, we treated glioma cells in vitro with anti-TNFRSF6B antibody and explored the effect of anti-TNFRSF6B antibody on the malignant phenotypes of glioma cell lines. 


\section{Materials and methods}

Cell culture and treatment with antibodies. Eight human malignant glioma cell lines (LN-18, LN-229, LN-308, LN-428, U87MG, U251MG, U373MG and D247MG) were purchased from the American Type Culture Collection (ATCC, Rockville, MD, USA). All cell lines were cultured in Dulbecco's modified essential medium, supplemented with $10 \%$ heat-inactivated fetal bovine serum (Invitrogen Corp., Grand Island, NY, USA), $2 \mathrm{mM}$ glutamine, gentamicin at $37^{\circ} \mathrm{C}$ in a humidified incubator with 5\% CO2. Previously, we showed that high expression of TNFRSF6B protein was detected in the supernatants and cells of U251MG, LN-308 and U87MG. Thus, these three cell lines were selected for further in vitro experiments in the present study. Experiments were performed on cells that had been transferred to serum-free medium $24 \mathrm{~h}$ previously, and were then supplemented with serum-free medium containing the indicated concentration of anti-TNFRSF6B neutralization antibody or hIgG1 (R\&D Systems, Inc. Minneapolis, MN USA) for the specified time. Blank controls were treated with an equivalent volume of serum-free DMEM medium. The concentration of anti-TNFRSF6B antibody was decided after a series of preliminary experiments showed a significant anti-tumor effect without cytotoxicity. All in vitro experiments were performed in triplicate.

Cell proliferation. Cell proliferation was assessed using a colorimetric tetrazolium (MTS) assay (CellTiter96 AQueous One Solution Cell Proliferation Assay G3580, Promega, Madison, WI, USA). Briefly, U251MG, LN-308 and U87MG cells in logarithmic growth-phase were collected, and $2.5 \times 10^{3}$ cells/well were dispersed in 96-well culture plates in $100 \mu \mathrm{l}$ volume. Then ascending concentrations of anti-TNFRSF6B neutralization antibody $(0.05,0.1,0.2,0.4,0.8,1.6,3.2$ and 6.4 $\mathrm{mg} / \mathrm{L}$ ) were delivered in corresponding wells. Each concentration above was regarded as one treated group while hIgG1 was in the negative control group and no antibody but only serum-free DMEM medium in the blank control group. Each group contained six parallel wells. Culture plates were then incubated at $37^{\circ} \mathrm{C}$ for $0,24,48,72,96$ and $120 \mathrm{~h}$, respectively. Following addition of $20 \mu \mathrm{l}$ of MTS reagent to each well, the plates were incubated for $2 \mathrm{~h}$ at $37^{\circ} \mathrm{C}$ in a humidified $5 \% \mathrm{CO} 2$ atmosphere, and the absorbance at $490 \mathrm{~nm}$ was recorded using a 96-well microplate reader (Scientific Multiskan MK3, Thermo Finland). The results were the mean of six wells and presented as the ratio of the absorbance of anti-TNFRSF6B antibody treated wells/absorbance of mock control $\times 100$.

Cell viability. To further support the data from the above MTS assay, cell viability was measured by fluorimetric detection of resorufin (CellTiter-Blue Cell Viability Assay, G8080, Promega, Madison, WI, USA). The procedure was according to the manufacturer instructions. The treatments and controls were as mentioned above. Fluorimetry (ex: 560 nm/em: 590 $\mathrm{nm}$ ) was using an FL600 fluorescence plate reader (Bio-Tek, Winooski, Vermont, USA). Fluorescence data were expressed as the fluorescence of treated sample/mock control $\times 100$.
Soft agar assay. U251MG cells $\left(2.5 \times 10^{4}\right)$ treated with anti-TNFRSF6B antibody were suspended in $2 \mathrm{ml}$ of medium (DMEM supplemented with 10\% FBS) containing 0.4\% agar. The cell mixture was then seeded on a layer of $1 \%$ bottom agar in 60-mm plate and allowed to grow for 4 weeks. After 4 weeks of incubation, colonies with more than 50 cells were stained by Giemsa and counted. A total of ten fields were counted for each plate.

Flow cytometry analysis of cell-cycle. U251MG cells $\left(2.5 \times 10^{5}\right)$ were plated into 6-well culture plates and treated with anti-TNFRSF6B antibody, hIgG1 and serum-free medium respectively for $96 \mathrm{~h}$. Treated cells were collected with trypsin, then were washed once with $4^{\circ} \mathrm{C}$ PBS, and fixed in cold $75 \%$ ethanol at $4^{\circ} \mathrm{C}$. Next, cells washed once again with $4^{\circ} \mathrm{C}$ PBS and re-suspended with PBS, then stained with $50 \mathrm{mg} /$ $\mathrm{ml}$ propidium iodide (PI, Sigma-Aldrich N.V. Bornem, Belgium) and $100 \mathrm{mg} / \mathrm{ml}$ RNase A solution (Genview, Carlsbad, CA) for $20 \mathrm{~min}$ at $37^{\circ} \mathrm{C}$ in dark. Stained cells were subjected to analysis immediately by flow cytometry. The proportion of cells in each phase of the cell cycle was determined by a BDFACScan for Quantitative Cell Analysis. Three independent measurements were made and averaged.

Western blot analysis. After being treated variously for the indicated timein 6-well-plate, the cells were washed with PBS and lysed in a buffer containing $80 \mathrm{mM}$ Tris- $\mathrm{HCl}$ (ph 6.8), 5\% SDS, 10\% glycerol, 5 mM EDTA (ph 8), 5\% 2-MercaptoEthanol, $0.2 \%$ Bromophenolblue, and $1 \mathrm{mM}$ phenylmethylsulfonyl fluoride. The lysates were centrifuged at $12,000 \times \mathrm{g}$ for $10 \mathrm{~min}$ at $4^{\circ} \mathrm{C}$ and boiled for $5 \mathrm{~min}$. Twentyfive microgram protein of each sample was subjected to SDS-PAGE ( $8 \%$ SDS-acrylamide gel) and separated proteins were transferred to hybond ECL nitrocellulose membranes (GE Healthcare Bio-sciences/Amersham, Diegem, Belgium) for $2 \mathrm{~h}$ at $100 \mathrm{~mA}$. The membrane was incubated with an antibody of TNFRSF6B (ab11930, Abcam), matrix metalloproteinase-2 (MMP-2, 4022, Cell Signaling Technology), phospho-ERK1/2 (pTpY185/187, Invitrogen), total-ERK1/2 (9102, Cell Signaling Technology), phospho-AKT/PKB (pS473, Invitrogen), total-AKT (9272, Cell Signaling Technology), or $\beta$-actin (AC-15, Sigma-Aldrich). Primary antibodies were detected with an HRP-conjugated secondary antibody (GE Healthcare Bio-sciences/Amersham, Diegem, Belgium) and finally the membranes were subjected to chemiluminescence detection assay (ECL Plus Western Blotting Detection Reagents, GE Healthcare Bio-sciences/ Amersham, Diegem, Belgium).

Caspase-3/7 activity detection. Caspase-3/7 activity was measured using a synthetic rhodamine labeled caspase-3/7 substrate (Apo-ONE Homogeneous Caspase-3/7 Assay, G7790, Promega, Madison, WI, USA) performed instantly after the detection of cell viability (described above) on the same wells, according to the instructions of the manufacturer. After incubation at room temperature for $60 \mathrm{~min}$, the fluorescence of each well was measured (ex: $499 \mathrm{~nm} / \mathrm{em}: 512 \mathrm{~nm}$ ), using a FL600 fluorescence plate reader (Bio-Tek, Winooski, 
Vermont, USA). Caspase-3/7 activity is expressed as fluorescence of treated sample/mock control $\times 100$.

Fluorescent microscopy evaluation of cell apoptosis and morphology. The effects of anti-TNFRSF6B antibody on apoptosis and nuclear morphology in the cells were evaluated by Hoechst 33342 (Sigma-Aldrich N.V. Bornem, Belgium) and PI double fluorescent chromatin staining. In brief, after treatment, cells were washed with ice-cold PBS and stained $15 \mathrm{~min}$ with Hoechst $33342(1 \mathrm{mg} / \mathrm{ml})$ and PI $(1 \mathrm{mg} / \mathrm{ml})$, and observed under fluorescence microscope (ZEISS Axiovert 25, Zaventem,
Belgium). Apoptosis and nuclear morphology were identified by condensation of nuclear chromatin and its fragmentation. This system determines the absolute number of viable cells (Hoechst 33342 positive/PI negative), early apoptotic cells (Hoechst 33342 positive/PI negative with blue fragmentations in the cells), late apoptotic cells (Hoechst 33342 positive/PI positive, with red fragmentations in the cells), necrotic cells (PI positive), and debris signals. Viable, apoptotic, and necrotic cells were counted in 10 different fields under the $200 \times$ vision in each well in three independent experiments by two persons
A
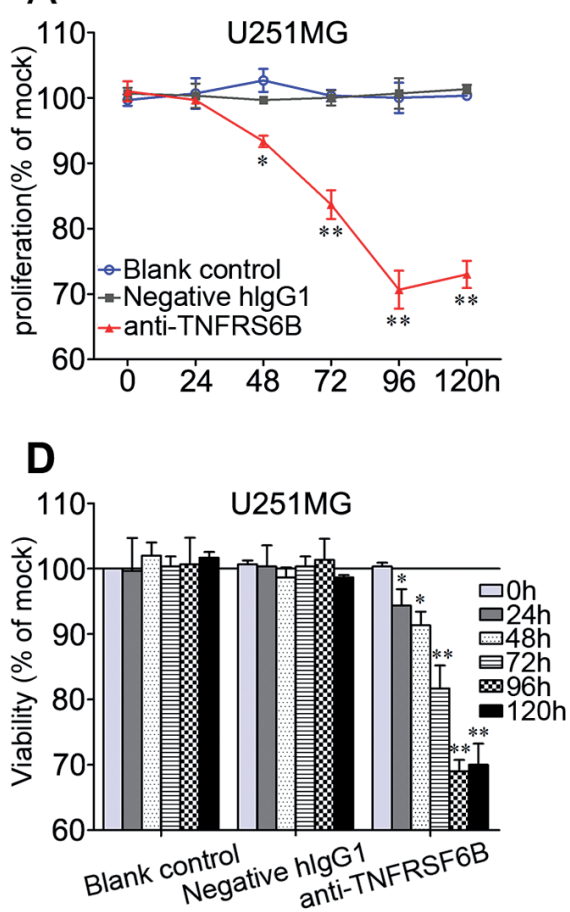

G

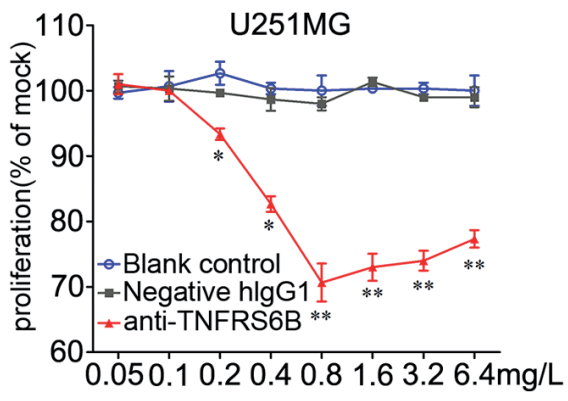

B

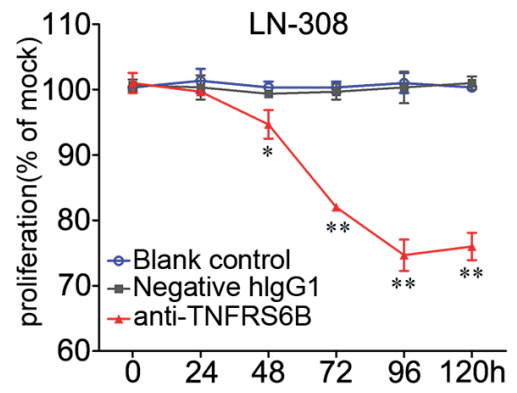

E

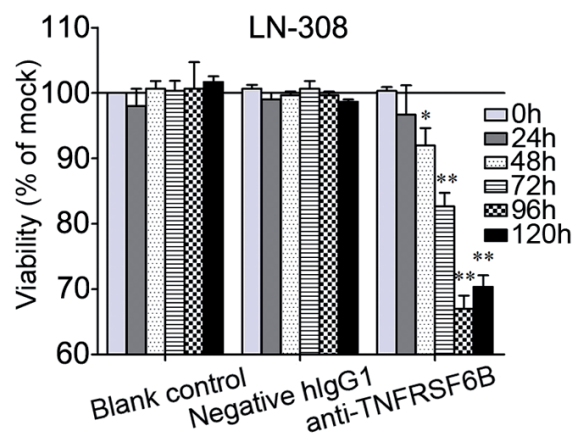

$\mathrm{H}$

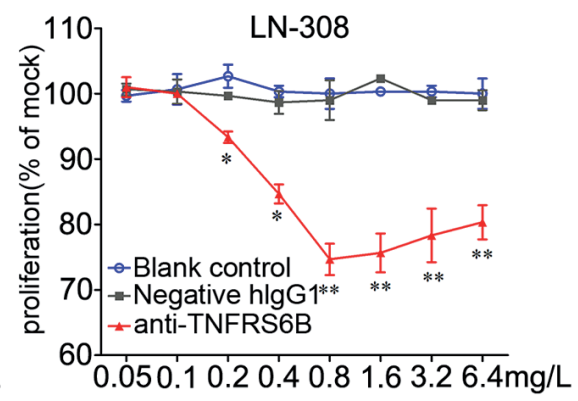

C

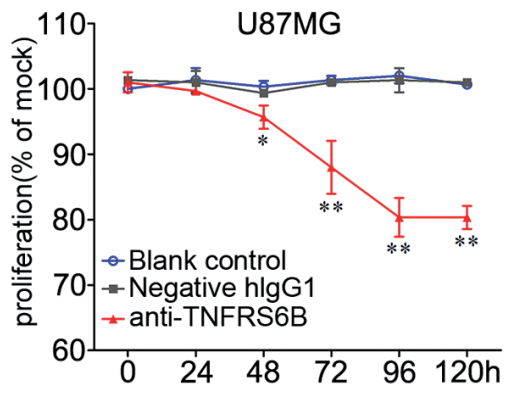

$\mathbf{F}$
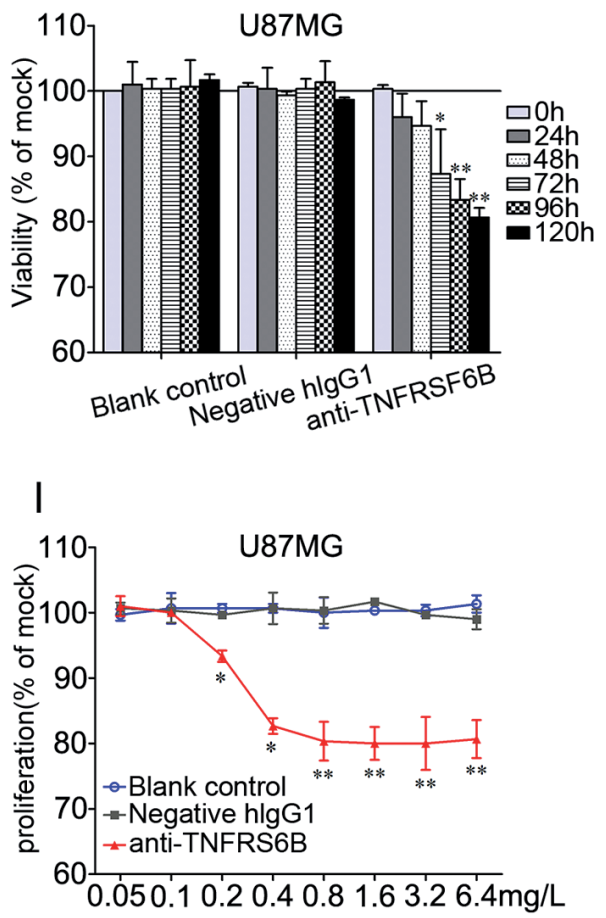

Figure 1. Effect of TNFRSF6B antibody on cell growth in glioma cell lines

U251MG, LN-308 and U87MG cells $\left(2.5 \times 10^{3}\right.$ cells per well in 96-well-plate) were cultured for $24 \mathrm{~h}$ and then treated with TNFRSF6B antibody and the negative hIgG1 control $(0.8 \mathrm{mg} / \mathrm{L})$ up to another $120 \mathrm{~h}$. Cell proliferation (U251MG (A), LN-308(B), U87MG(C)) and cell viability (U251MG (D), LN308(E), U87MG(F)) were assessed per day with MTS assay (CellTiter96 Aqueous One Solution Cell Proliferation Assay) and fluorimetric detection of resorufin (CellTiter-Blue Cell Viability Assay), respectively. The same amount of these cell lines $\left(2.5 \times 10^{3}\right.$ cells per well in 96 -well-plate) were cultured as above with increasing concentrations of TNFRSF6B antibody and the negative hIgG1 control for 96h (0-6.4 mg/L). Cell proliferation (U251MG (G), LN-308(H), U87MG(I)) for each dosage was monitored with MTS assay. ${ }^{\star} \mathbf{P}<0.05,{ }^{\star *} \mathbf{P}<0.01$, compared to blank and negative controls at the same time point or with the equal concentration. 
and the average result was compared to the mock control. Apoptotic cell numbers from different treatments were compared by being normalized to their viable cell numbers.

Statistical analysis. Statistical analysis was performed using SPSS 20.0 software for Windows (Munich, Germany). Data of inhibitory rate from cell growth assays, numbers of colonies from soft agar assay, proportion of cell cycles from flow cytometry and apoptotic data were expressed as mean \pm $\mathrm{SD}$. Differences between groups were examined for statistical significance using analysis of variance (ANOVA) and Student's $t$-test. A $P$ value of less than 0.05 was considered statistically significant.

\section{Results}

Cell growth inhibitory effect of anti-TNFRSF6B neutralization antibody. Our previous work detected overexpression of TNFRSF6B protein in all 3 cell lines in the present study [14]. The effect of anti-TNFRSF6B antibody on the growth of glioma cells was evaluated using several independent assays, including MTS tetrazolium assay, fluorimetric resorufin viability assay, Hoechst 33342/PI double fluorescent chromatin staining, soft agar colony assay and flow cytometry, respectively. MTS tetrazolium assay revealed that cell proliferation was not influenced in the hIgG1 controls in all 3 cell lines tested. However, after treatment with anti-TNFRSF6B antibody, a large reduction in proliferation was examined at 48 , 72 and $96 \mathrm{~h}$ in all three cell lines tested and the most potent effect occurred at 96h (Figure1A, Figure1B, Figure1C). In all 3 cell lines, anti-TNFRSF6B antibody offered the most powerful impact on U251MG cells, with the proliferation rate of $70.667 \pm$ $5.033 \%$ at $96 \mathrm{~h}$ post treatment. To confirm these results, the effect on cell viability was assessed by using a fluorimetric resorufin viability assay (Figure1D, Figure1E, Figure1F) and Hoechst 33342/PI double fluorescent chromatin staining (data not shown), which largely mirrored the results from MTS assay and the cell growth inhibitory effect of anti-TNFRSF6B antibody showed a time dependent manner on glioma cells.
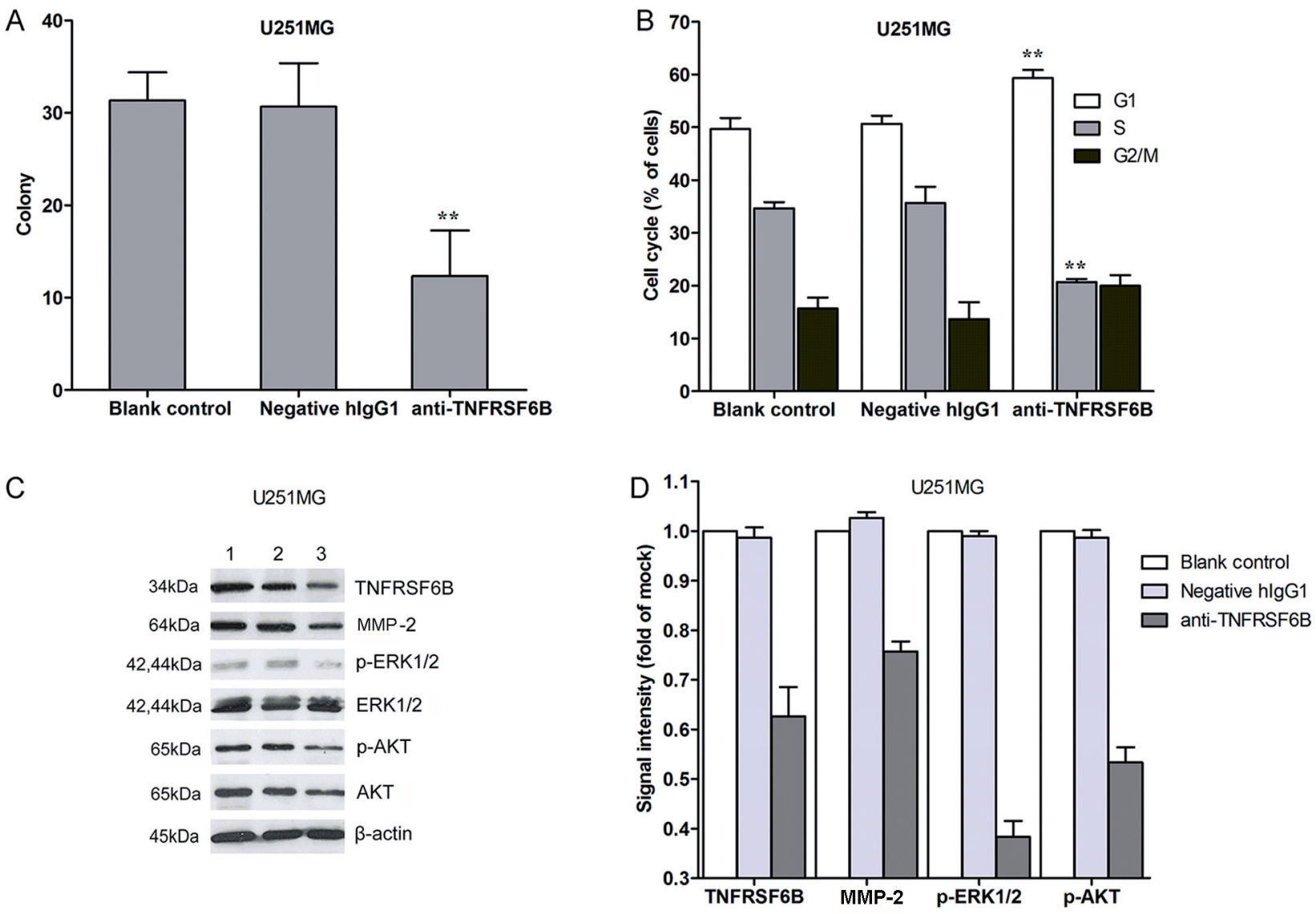

Figure 2 Effect of TNFRSF6B antibody on colony, cell cycle and relevant downstream pathways in glioma U251MG cells

U251MG cells $\left(2.5 \times 10^{4}\right.$ cells per well in $60-\mathrm{mm}$-plate $)$ were cultured for $24 \mathrm{~h}$ then transfected with TNFRSF6B antibody and the negative hIgG1 control $(0.8 \mathrm{mg} / \mathrm{L})$ for 3 weeks to check the ability of colony formation by using soft agar assay (A). U251MG cells (2.5×10 5 cells per well in 6-well-plate) were cultured for $24 \mathrm{~h}$ then transfected with TNFRSF6B antibody and the negative hIgG1 control $(0.8 \mathrm{mg} / \mathrm{L})$ for $96 \mathrm{~h}$. Cell cycle was analyzed with flow cytometry (B) and the relevant downstream proteins were examined by using western blot (C, D). Antibodies included: TNFRSF6B, MMP-2, phospho-ERK1/2 (p-ERK1/2), total-ERK1/2 (t-ERK1/2), p-AKT, t-AKT and $\beta$-Actin. 1: Blank control; 2: Negative hIgG1; 3: anti- TNFRSF6B antibody. 
The effect of anti-TNFRSF6B antibody on cell growth suppression showed also a dose dependent manner detected by MTS tetrazolium assay (Figure1G, Figure1H, Figure1I), fluorimetric resorufin viability assay and Hoechst 33342/ PI staining (data not shown). Furthermore, soft agar colony formation showed that in the group treated by anti-TNFRSF6B antibody, colony rate was $12.333 \pm 4.933 \%$, significantly lower than that in the blank $(31.333 \pm 3.055 \%)$ and hIgG1 groups $(30.667 \pm 4.726 \%, P<0.001)$ in glioma U251MG cells (Figure
2A, data of LN-308 and U87MG not shown ). Growth inhibition by anti-TNFRSF6B antibody in the HepG2 cells was accompanied by cell cycle G1/S arrest, which was indicated by a decrease in DNA synthesis and retardation of cell growth. Anti-TNFRSF6B antibody inhibited DNA synthesis and increased the percentage of U251MG cells in G0-G1 phase. After 96h treatment, the percentage of G0-G1 phase cells was $59.333 \pm 1.528 \%$ in anti-TNFRSF6B antibody treated group compared with $49.667 \pm 2.082 \%$ in blank and $50.667 \pm 1.528 \%$

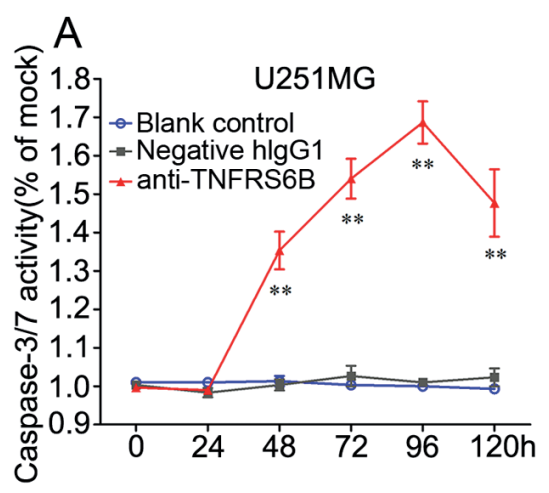

D
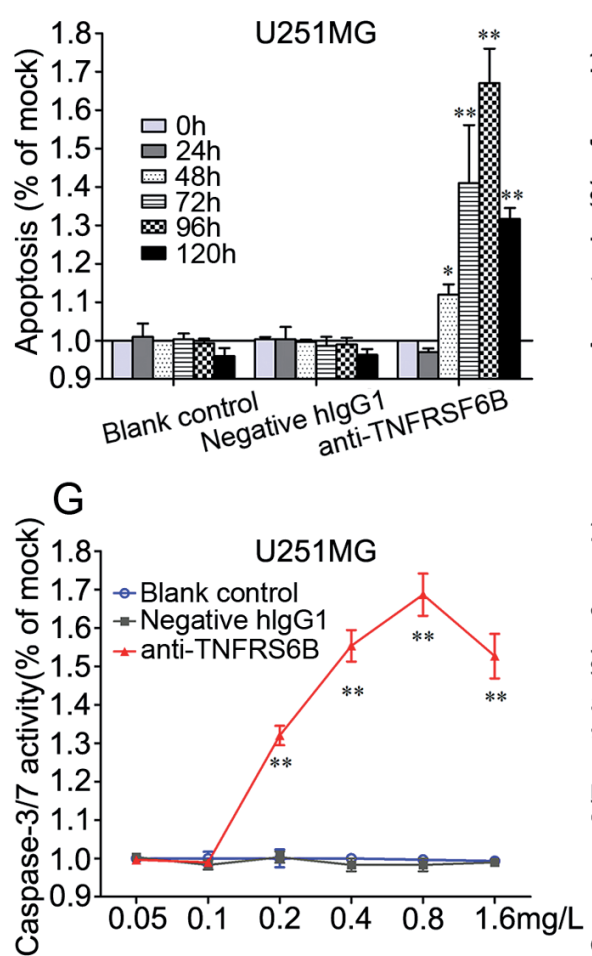

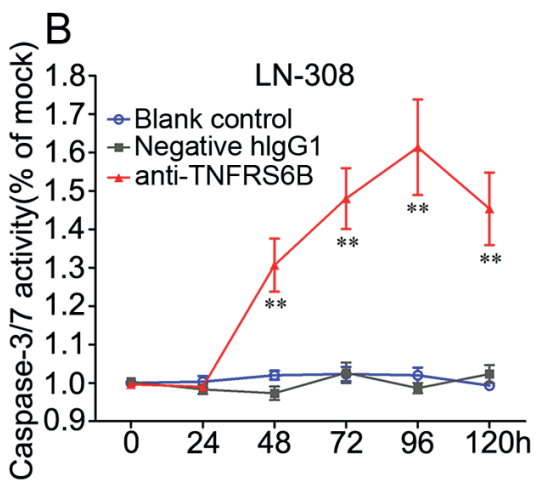

E
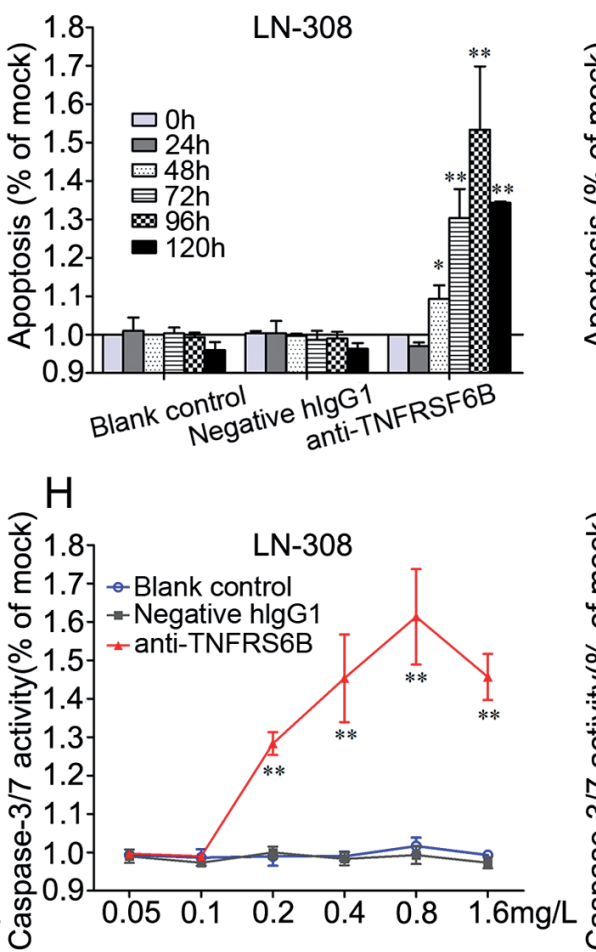
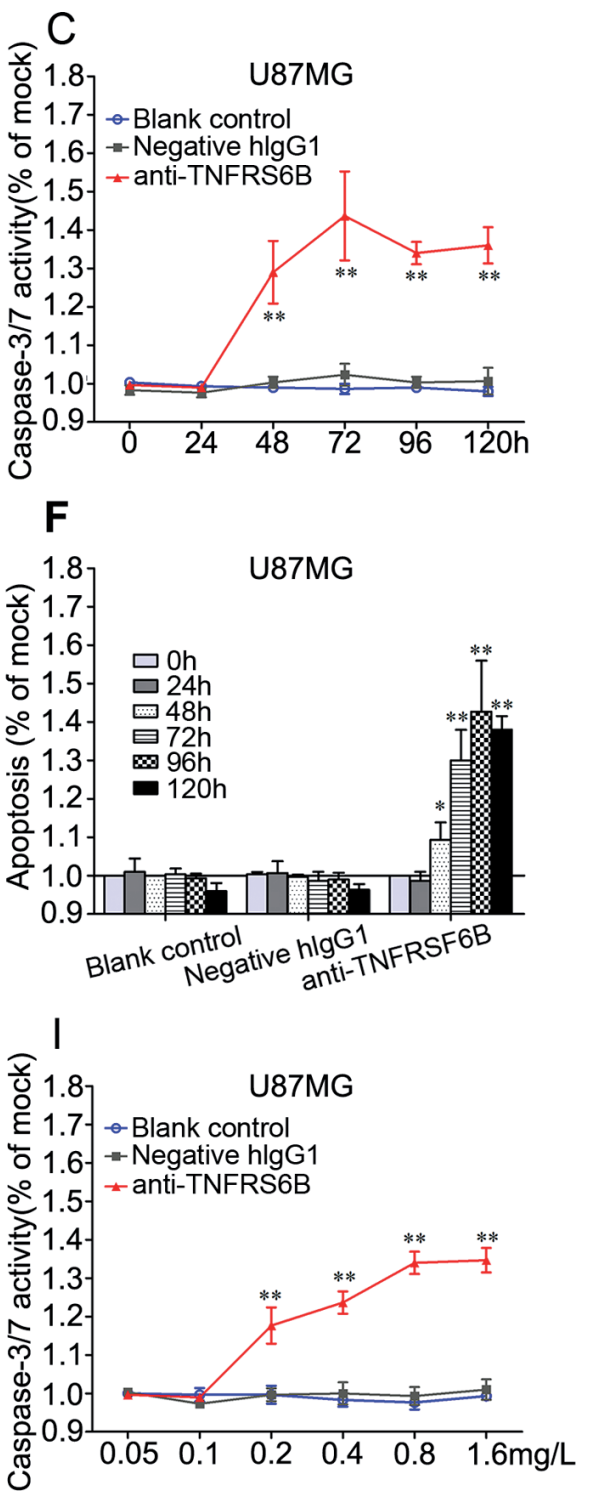

Figure 3. Effect of TNFRSF6B antibody on cellular apoptosis in glioma cell lines

U251MG, LN-308 and U87MG cells $\left(2.5 \times 10^{3}\right.$ cells per well in 96-well-plate) were cultured for $24 \mathrm{~h}$ and then treated with TNFRSF6B antibody and the negative hIgG1 control $(0.8 \mathrm{mg} / \mathrm{L})$ up to another $120 \mathrm{~h}$. Caspase-3/7 activity (U251 MG (A), LN-308(B), U87MG(C)) and apoptosis (U251 MG (D), LN-308(E), U87MG(F)) were assessed per day with Apo-ONE Homogeneous Caspase-3/7 Assay and Hoechst 33342/ propidium iodide (PI) double fluorescent chromatin staining, respectively. The same amount of these cell lines $\left(2.5 \times 10^{3}\right.$ cells per well in 96 -well-plate) were cultured as above with increasing concentrations of TNFRSF6B antibody and the negative hIgG1 control for 96h (0-6.4 mg/L). Caspase-3/7 activity (U251 MG (G), LN-308(H), U87MG(I)) for each dosage was monitored with Apo-ONE Homogeneous Caspase-3/7 Assay. ${ }^{\star} \mathrm{P}<0.05$, ${ }^{\star *} \mathrm{P}<0.01$, compared to blank and negative controls at the same time point or with the equal concentration. 
in hIgG1 control. This increase was coupled with the reduced percentage of cells in S phase. The percentage of $S$ phase cells was $20.667 \pm 0.577 \%$ in anti-TNFRSF6B antibody treated group compared with $34.667 \pm 1.155 \%$ in blank and $35.667 \pm 3.055 \%$ in hIgG1 control. Similar but slightly weaker effect of antiTNFRSF6B antibody on the cell cycle was also observed in other two cell lines LN-308 and U87MG (data now shown).

Apoptosis induced by anti-TNFRSF6B antibody. To validate whether anti-TNFRSF6B antibody was able to influence apoptosis in glioma cells, the CellTiter-Blue assay was multiplexed with a fluorescent caspase-3/7 assay. The results showed that with anti-TNFRSF6B antibody, caspase- $3 / 7$ activity was markedly enhanced in all 3 glioma cell lines tested with a time (Figure 3A, Figure 3B, Figure 3C) and dose dependent manner (Figure 3G, Figure 3H, Figure 3I). Similar to the result of cell growth, the most powerful effect of anti-TNFRSF6B antibody on caspase activity occurred in U251MG cells, with the dose of $0.8 \mathrm{mg} / \mathrm{L} 96 \mathrm{~h}$ post treatment, as 1.687 folds of the mock control (Figure 3A, Figure 3G). The effect on apoptosis was confirmed microscopically by Hoechst 33342 and PI double fluorescent staining (Figure 3D, Figure 3E, Figure 3F and Figure 4).

Contribution of anti-TNFRSF6B antibody in relevant cellular signaling. To investigate the contribution of antiTNFRSF6B antibody in the regulation of cellular signaling, we examined the signaling of MMP-2, ERK and AKT pathways by using western blot, which are related to cell survival, apoptosis, invasion and metastasis. These pathways were influenced little with hIgG1 control. However, the MMP-2, phospho-ERK1/2 and phospho-AKT were down-regulated by anti-TNFRSF6B antibody $96 \mathrm{~h}$ post treatment (Figure 2C, Figure 2D).

\section{Discussion}

TNFRSF6B has been regarded as an oncogene for some malignancies, including glioma [14-16]. Previously, we de- tected higher expression of TNFRSF6B in the supernatants of different glioma cell lines and the freshly primary cultured glioma cells, as well as in glioma tissues [14]. We also found that the overexpression of TNFRSF6B in glioma tissues was positively correlated with the differentiation of glioma cells, as evaluated by morphology and glial fibrillary acidic protein (GFAP) staining [14]. To further explore the function of TNFRSF6B on the malignant phenotypes of glioma cells and to probe the potential application of anti-TNFRSF6B antibody for the treatment of glioma, we performed a series of in vitro experiments.

Previously, we found that in glioma tissues, TNFRSF6B expression was positive correlated with the labelling index of Ki-67 and PCNA, which demonstrated that in the higher actively proliferated gliomas, TNFRSF6B showed stronger expression. This correlation between TNFRSF6B and growth of glioma cells was further tested in vitro in the current study. The results showed that anti-TNFRSF6B antibody decelerated the cell growth in all cell lines tested (U251MG, LN-308 and U87MG) with a time and dose-dependent manner by using four independent assays (MTS tetrazolium assay, fluorimetric resorufin viability assay, Hoechst 33342/ PI staining and soft agar colony assay). Surprisingly, in all the 3 cell lines tested (U251MG, LN-308 and U87MG), high dosage of antibody $(>0.8 \mathrm{mg} / \mathrm{L})$ did not further inhibit cell growth. We think that the heterogeneity of glioma cells could partially explain the phenomenon. Some glioma cells could be sensitive to antiTNFRSF6B antibody treatment, thus a decreasing pattern from $0.05-0.8 \mathrm{mg} / \mathrm{L}$ was observed. With the dosage of 0.8 $\mathrm{mg} / \mathrm{L}$, all glioma cells sensitive to anti-TNFRSF6B antibody were blocked and those resistant to anti-TNFRSF6B antibody were still growing. However, no statistical difference was found between all high dosages of antibody $(0.8,1.6,3.2$ and 6.4 $\mathrm{mg} / \mathrm{L}$, data not shown). Moreover, flow cytometry showed that inhibition of cell growth in glioma cells by anti-TNFRSF6B

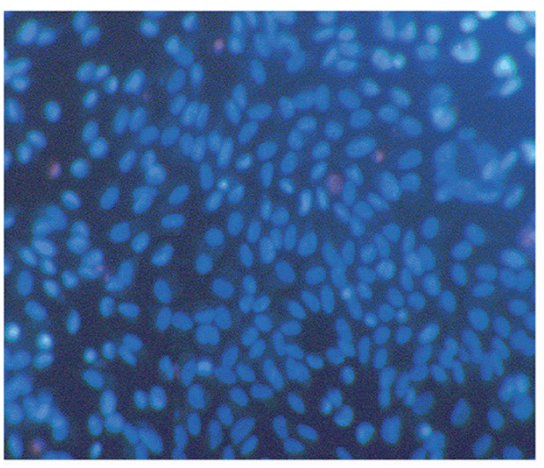

Blank control

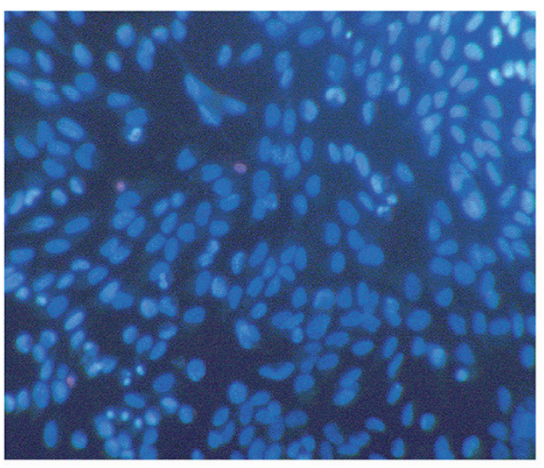

Negative hlgG1

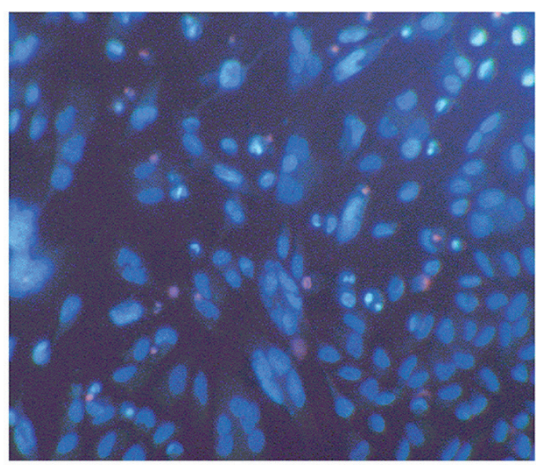

anti-TNFRSF6B

Figure 4 TNFRSF6B antibody suppressed cell growth and induced apoptosis with Hoechst 33342/ propidium iodide (PI) double fluorescent chromatin staining

U251MG cells $\left(2.5 \times 10^{3}\right.$ cells per well in 96 -well-plate $)$ were cultured for $24 \mathrm{~h}$ then transfected with TNFRSF6B antibody and the negative hIgG1 control $(0.8 \mathrm{mg} / \mathrm{L})$ up to another $96 \mathrm{~h}$. Hoechst $33342 /$ PI double fluorescent chromatin staining was performed to examine the effect of TNFRSF6 antibody on the cell growth and apoptosis in U251MG cells. $\times 200$. 
antibody was accompanied by retardation of G1/S transition. This helps to explain the aggressive capacity of fast growing of glioma cells in clinic.

TNFRSF6B has been reported to assist tumor cells to gain survival advantage by inhibiting apoptosis due to competitive inhibition of the binding of TNF to TNFRs. Hence, we were interested about the role of anti-TNFRSF6B antibody on the apoptosis of glioma cells in vitro. We found that antiTNFRSF6B antibody could enhance the caspase-3/7 activity and induce apoptosis in glioma cell lines, also in a time and dose-dependency. To explore the possible molecular mechanism of the contribution of anti-TNFRSF6B antibody in glioma cells, we performed western blot to determine the influence of anti-TNFRSF6B antibody on MMP-2, ERK1/2 and AKT. MMP-2 is a proteolytic enzyme that can degrade extracellular matrix components and plays an important role in tumorigenesis, invasion and metastasis of cancers [17-24] and it has been reported that in esophageal cancer tissues, TNFRSF6B expression is positively correlated to MMP-2 [19]. The activation of ERK and AKT pathways also represent the ability of survival, proliferation and apoptosis in cancers. The results from western blot showed that the function of antiTNFRSF6B antibody on cell growth inhibition and apoptosis induction could partially be explained by the downregulation of MMP-2 and the downstream pathways, especially ERK1/2 and AKT in glioma cells.

Taken together, our study showed that anti-TNFRSF6B antibody could suppress cell growth and induce apoptosis in human glioma cells in vitro. This anti-tumor effect is due to G1/S arrest in cell cycle and downregulation of MMP-2, p-ERK1/2 and $\mathrm{p}$-AKT. To our knowledge, this is the first report on the effect of anti-TNFRSF6B antibody on human glioma cells. The suppressive role of anti-TNFRSF6B antibody on the malignant phenotypes of glioma cells makes it tempting to develop novel therapeutical strategies. The application of anti-TNFRSF6B antibody might thus be a promising approach to glioma therapies in the future, for both in vivo studies and clinic trials.

Acknowledgements: The study was supported by the Fund of Ministry of Education and Scientific Research of Guangxi, China (200710LX062).

\section{References}

[1] KOVAR JL, CURTIS E, OTHMAN SF, SIMPSON MA, OLIVE DM. Characterization of IRDye $800 \mathrm{CW}$ chlorotoxin as a targeting agent for brain tumors. Analytical biochemistry 2013, 440: 212-219. http://dx.doi.org/10.1016/j. ab.2013.05.013

[2] KAO HW, CHIANG SW, CHUNG HW, TSAI FY, CHEN CY. Advanced MR imaging of gliomas: an update. BioMed research international 2013, 2013: 970586.

[3] CHANG L, SU J, JIA X, REN H. Treating malignant glioma in Chinese patients: update on temozolomide. OncoTargets and therapy $2014,7: 235-244$.
[4] WANG YX, FAN K, TAO DB, DONG X. Association between genetic polymorphism of xrccl gene and risk of Glioma in a Chinese population. Asian Pacific journal of cancer prevention : APJCP 2013, 14: 5957-5960.

[5] CHOI J, LEE EY, SHIN KJ, MINN YK, KIM J, ET AL. IDH1 mutation analysis in low cellularity specimen: a limitation of diagnostic accuracy and a proposal for the diagnostic procedure. Pathology, research and practice 2013, 209: 284-290. http://dx.doi.org/10.1016/j.prp.2013.02.010

[6] MORISHIGE T, YOSHIOKA Y, INAKURA H, TANABE A, YAO X, et al. Creation of a lysine-deficient LIGHT mutant with the capacity for site-specific PEGylation and low affinity for a decoy receptor. Biochemical and biophysical research communications 2010, 393: 888-893. http://dx.doi. org/10.1016/j.bbrc.2010.02.119

[7] CONNOR JP, FELDER M, KAPUR A, ONUJIOGU N. DcR3 binds to ovarian cancer via heparan sulfate proteoglycans and modulates tumor cells response to platinum with corresponding alteration in the expression of BRCA1. BMC cancer 2012, 12: 176. http://dx.doi.org/10.1186/1471-2407-12-176

[8] WANG W, ZHANG M, SUN W, YANG S, SU Y, et al. Reduction of Decoy Receptor 3 Enhances TRAIL-Mediated Apoptosis in Pancreatic Cancer. PloS one 2013, 8: e74272. http://dx.doi.org/10.1371/journal.pone.0074272

[9] YANG D, FAN X, YIN P, WEN Q, YAN F, et al. Significance of decoy receptor 3 (Dcr3) and external-signal regulated kinase 1/2 (Erk1/2) in gastric cancer. BMC immunology 2012, 13: 28. http://dx.doi.org/10.1186/1471-2172-13-28

[10] CHEN G, LUO D. Expression of decoy receptor 3 in liver tissue microarrays. The National medical journal of India 2008 , 21: 275-278.

[11] CHEN G, LUO D. Over-expression of decoy receptor 3 in gastric precancerous lesions and carcinoma. Upsala journal of medical sciences 2008, 113: 297-304. http://dx.doi. org/10.3109/2000-1967-240

[12] CHEN G, RONG M, LUO D. TNFRSF6B neutralization antibody inhibits proliferation and induces apoptosis in hepatocellular carcinoma cell. Pathology, research and practice 2010, 206: 631-641. http://dx.doi.org/10.1016/j. prp.2010.05.011

[13] YANG M, CHEN G, DANG Y, LUO D. Significance of decoy receptor 3 in sera of hepatocellular carcinoma patients. Upsala journal of medical sciences 2010, 115: 232-237. http://dx.doi. org/10.3109/03009734.2010.516410

[14] HUANG S, CHEN G, DANG Y, CHEN LH. Overexpression of DcR3 and its significance on tumor cell differentiation and proliferation in glioma. TheScientificWorldJournal 2014, 2014: 605236.

[15] ROTH W, ISENMANN S, NAKAMURA M, PLATTEN M, WICK W, et al. Soluble decoy receptor 3 is expressed by malignant gliomas and suppresses CD95 ligand-induced apoptosis and chemotaxis. Cancer research 2001, 61: 2759-2765.

[16] ARAKAWA Y, TACHIBANA O, HASEGAWA M, MIYAMORI T, YAMASHITA J, et al. Frequent gene amplification and overexpression of decoy receptor 3 in glioblastoma. Acta neuropathologica 2005, 109: 294-298. http://dx.doi. org/10.1007/s00401-004-0956-6 
[17] XIN YH, BIAN BS, YANG XJ, CUI W, CUI HJ, et al. POU5F1 enhances the invasiveness of cancer stem-like cells in lung adenocarcinoma by upregulation of MMP-2 expression. PloS one 2013, 8: e83373. http://dx.doi.org/10.1371/journal. pone. 0083373

[18] GOU X, CHEN H, JIN F, WU W, LI Y, et al. Expressions of CD147, MMP-2 and MMP-9 in Laryngeal Carcinoma and its Correlation with Poor Prognosis. Pathology oncology research 2014, 20: 475-481. http://dx.doi.org/10.1007/s12253-013$\underline{9720-3}$

[19] AO R, DU YQ, WANG Y, CHEN YS, WANG BY. MMP-2 and DcR3 expression in esophageal cancer tissue and correlation with patient survival. International journal of clinical and experimental medicine 2013, 6: 700-705.

[20] JACOB-FERREIRA AL, KONDO MY, BARAL PK, JAMES MN, HOLT A, et al. Phosphorylation status of $72 \mathrm{kDa}$ MMP-2 determines its structure and activity in response to peroxynitrite. PloS one 2013, 8: e71794. http://dx.doi.org/10.1371/ journal.pone.0071794

[21] SEO KW, LEE SJ, KIM YH, BAE JU, PARK SY, et al. Mechanical stretch increases MMP-2 production in vascular smooth muscle cells via activation of PDGFR-beta/Akt signaling pathway. PloS one 2013, 8: e70437. http://dx.doi.org/10.1371/ journal.pone.0070437

[22] KORKMAZ KS, DE ROOIJ BJ, VAN HOEK B, JANSE M, COENRAAD MJ, et al. MMP-2 is a disease-modifying gene in primary sclerosing cholangitis. Liver international : official journal of the International Association for the Study of the Liver 2014, 34: 274-280.

[23] LEE H, CHANG KW, YANG HY, LIN PW, CHEN SU, et al. MT1-MMP regulates MMP-2 expression and angiogenesis-related functions in human umbilical vein endothelial cells. Biochemical and biophysical research communications 2013, 437: 232-238. http://dx.doi. org/10.1016/j.bbrc.2013.06.046

[24] WU HM, WANG HS, HUANG HY, LAI CH, LEE CL, et al. Gonadotropin-releasing hormone type II (GnRH-II) agonist regulates the invasiveness of endometrial cancer cells through the GnRH-I receptor and mitogen-activated protein kinase (MAPK)-dependent activation of matrix metalloproteinase (MMP)-2. BMC cancer 2013, 13: 300. http://dx.doi. org/10.1186/1471-2407-13-300 